\title{
ESTIMATES OF THE CACCIOPPOLI-SCHAUDER TYPE IN WEIGHTED FUNCTION SPACES
}

\author{
GIOVANNI MARIA TROIANIELLO
}

\begin{abstract}
We deal with imbeddings of certain weighted function spaces as well as with the corresponding norm estimates for solutions to second order elliptic problems. We redemonstrate some results of Gilbarg and Hörmander by a technique, entirely different from theirs, which enables us to cover a range of parameters excluded by them.
\end{abstract}

\section{INTRODUCTION}

Consider the Dirichlet problem

$$
L u=f \text { in } \Omega,\left.\quad u\right|_{\partial \Omega}=\phi,
$$

where $\Omega$ is a bounded open subset of $\mathbf{R}^{N}, \partial \Omega$ its boundary, and $L$ a linear second order uniformly elliptic differential operator with coefficients defined on $\bar{\Omega}$. The classical Caccioppoli-Schauder approach to $(0.1)$ provides, , under suitable regularity assumptions about $\partial \Omega$ and the coefficients of $L$, a priori bounds on norms $|u|_{C^{m, \delta}(\bar{\Omega})}$ when $f$ is in $C^{m-2, \delta}(\bar{\Omega})$ and $\phi$ in $C^{m, \delta}(\partial \Omega)$, the space of traces on $\partial \Omega$ of functions from $C^{m, \delta}(\bar{\Omega})$ (see the notations in $\S 1$ ). Here $m=2,3, \ldots$ and $0<\delta<1$ : well-known examples show that for the validity of the theory it is essential to exclude the values $\delta=0$ and $\delta=1$, hence to require

$$
m+\delta \notin \mathbf{N} .
$$

What happens now if we weaken our assumption about $\phi$ by requiring that it belong to $C^{m^{\prime}}, \delta^{\prime}(\partial \Omega)$ for some $m^{\prime}=0,1, \ldots$ and $\delta^{\prime} \in[0,1]$ such that $\alpha=m+\delta-\left(m^{\prime}+\delta^{\prime}\right)>0$ ? This question was tackled by Gilbarg and Hörmander [7], who (to put it roughly) showed that $\left[\operatorname{dist}\left(\partial \Omega, \partial \boldsymbol{\Omega}^{\prime}\right)\right]^{\alpha}|u|_{C^{m, \delta}\left(\bar{\Omega}^{\prime}\right)}$ is then bounded independently of $\Omega^{\prime} \Subset \Omega$ under the assumptions $(0.2)$,

$$
m+\delta-\alpha>0
$$

-which is natural for the problem at hand-and

$$
m+\delta-\alpha \notin \mathbf{N} .
$$

Received by the editors July 25, 1989 and, in revised form, March 23, 1990.

1980 Mathematics Subject Classification (1985 Revision). Primary 35D10, 35J25; Secondary 35B45, 35B65, 46E35. 
Note that, for what correspondingly concerns $f$, the natural regularity requirement is now only that $\left[\operatorname{dist}\left(\partial \Omega, \partial \Omega^{\prime}\right)\right]^{\alpha}|f|_{C^{m-2, \delta}\left(\bar{\Omega}^{\prime}\right)}$ be bounded independently of $\Omega^{\prime} \Subset \Omega$.

Let us give an idea of the motivations of (0.4).

We begin by sketchily reviewing the classical method through which the crucial estimates for solutions of $(0.1)$ are reduced to their counterparts for functions $u$ having compact supports and satisfying

$$
\Delta u=f \text { for } x_{N}>0, \quad u=0 \text { for } x_{N}=0 .
$$

First of all, a partition of unity and local changes of coordinates which flatten (portions of) the boundary show that it suffices to investigate $(0.1)$ when $\Omega$ is replaced by the half-space where $x_{N}>0, \partial \Omega$ by the hyperplane where $x_{N}=0$, and $u$ has compact support. Next, a local perturbation argument leads from the general case of variable coefficients to that of $L=\Delta$. Finally, a suitable extension of $\phi$ from the hyperplane to the entire half-space provides the equivalence between the previous inhomogeneous problem and $(0.5)$ (with a free term $f$ correspondingly modified, although within the original function class).

The three steps above are performed, in the optimal generality of assumptions about the regularity of $\partial \Omega$ and the coefficients of $L$, respectively by Theorem 5.1, Proposition 4.3 and Lemma 2.3 of [7]. For what concerns $(0.5)$ the approach of [7] (in Theorem 3.1) utilizes the integral representation of $u$ by means of Green's function. This makes it necessary to bound a term

$$
\sup _{x_{N}>0} x_{N}^{-(m+\delta-\alpha)}|u(x)|
$$

(where, we recall, $u=0$ for $x_{N}=0$ ), and it is at this point that $(0.4)$ comes to play an essential role.

In the present paper we develop a different approach to $(0.5)$. We put weights into Campanato, instead of Hölder, function spaces, and utilize variational estimates instead of Green's function. This enables us to redemonstrate the bound provided by Theorem 3.1 of [7] under the restrictions (0.2) (as in the Caccioppoli-Schauder theory for $\alpha=0$ ) and (0.3), without requiring (0.4). It is the case when the latter is violated, of course, that poses greater difficulties in the proofs. With our techniques we can also tackle the case when $(0.2)$ is violated; for some elucidations concerning the scope of the results we obtain then, see Remark 2 after the proof of Theorem 2.

It is probably worth mentioning here that the different roles of, as well as the interplay between, the parameters $\alpha$ and $\delta$ lead us to introduce, in the next section, the notation $C_{\alpha}^{m, \delta}$ for the function classes denoted in [7] by $H_{b}^{(a)}$ with $b=m+\delta, a+b=\alpha$. The parameter $m+\delta-\alpha$ measures, so to speak, the maximal global regularity which is attained without weight: when $\alpha=h+\delta$ for some $h=0, \ldots, m$ such a regularity does not go as far as $C^{m-h, 0}$ (see Lemmas 1.3 and 1.4 below).

We do not work our way from $(0.5)$ back to $(0.1)$. When (0.2) holds, that is, when $0<\delta<1$, this would amount to our verifying that the corresponding steps of [7] can be repeated without requiring $(0.4)$. When $(0.2)$ is violated, say for $\delta=0$, the regularity assumptions about $\partial \Omega$ and the coefficients of 
$L$ would be those corresponding to the case when $\delta$ is instead some positive number, no matter how small.

The results of the present article were announced in [13].

\section{FunCtion SPACES}

Some basic notations:

$x \equiv\left(x_{1}, \ldots, x_{N}\right) \equiv\left(x^{\prime}, x_{N}\right)$, the variable point of $\mathbf{R}^{N}$,

$\nabla \equiv\left(\partial / \partial x_{1}, \ldots, \partial / \partial x_{N}\right)$,

$D^{\mu} \equiv \partial^{|\mu|} / \partial x_{1}^{\mu_{1}}, \ldots, \partial x_{N}^{\mu_{N}}$ when $\mu$ is a multi-index $\left(\mu_{1}, \ldots, \mu_{N}\right)$ of nonnegative integers, $|\mu| \equiv \mu_{1}+\cdots+\mu_{N}$,

$B_{r}(y) \equiv\left\{x \in \mathbf{R}^{N}|| x-y \mid<r\right\}, B \equiv B_{1}(0)$,

$B_{r}^{+}(y) \equiv\left\{x \in B_{r}(y) \mid x_{N}>y_{N}\right\}, B_{r}^{+} \equiv B_{r}^{+}(0), B^{+} \equiv B_{1}^{+}$,

$S_{r}^{0}(y) \equiv\left\{x \in B_{r}(y) \mid x_{N}=y_{N}\right\}, S_{r} \equiv S_{r}^{0}(0), S^{0} \equiv S_{1}^{0}$.

Let $\omega$ be a bounded open subset of $\mathbf{R}^{N}$. If $k$ is a nonnegative integer $C^{k}(\bar{\omega})$ denotes the space of functions $u: \omega \rightarrow \mathbf{R}$ having uniformly continuous derivatives of order $\leq k$, normed by

$$
|u|_{C^{k}(\bar{\omega})} \equiv \sum_{|\mu| \leq k} \sup _{\omega}\left|D^{\mu} u\right| .
$$

If in addition $0<\delta \leq 1 C^{k, \delta}(\bar{\omega})$ denotes the space of functions $u$ such that

$$
|u|_{C^{k, \delta}(\bar{\omega})} \equiv|u|_{C^{k}(\bar{\omega})}+\sum_{|\mu|=k} \sup _{\substack{x, y \in \omega \\ x \neq y}} \frac{\left|D^{\mu} u(x)-D^{\mu} u(y)\right|}{|x-y|^{\delta}}
$$

is finite; instead of adopting $C^{k, 0}(\bar{\omega}) \equiv C^{k}(\bar{\omega})$ as is usual in the literature, here we find it convenient to have $C^{k, 0}(\bar{\omega}) \equiv C^{k-1,1}(\bar{\omega})$ for $k \in \mathbf{N}$ and $C^{0,0} \equiv L^{\infty}(\omega)$, the space of measurable functions $u: \omega \rightarrow \mathbf{R}$ such that

$$
|u|_{L^{\infty}(\omega)} \equiv \operatorname{ess} \sup _{\omega}|u|
$$

is finite. For $1 \leq p<\infty L^{p}(\omega)$ denotes the Lebesgue space of exponent $p$, normed by

$$
|u|_{L^{p}(\omega)} \equiv\left(\int_{\omega}|u|^{p} d x\right)^{1 / p}
$$

$H^{k, p}(\omega)$ the Sobolev space of order $k$ and exponent $p$, normed by

$$
|u|_{H^{k, p}(\omega)} \equiv\left(\sum_{|\mu| \leq k}\left|D^{\mu} u\right|_{L^{p}(\omega)}^{p}\right)^{1 / p}
$$

and $H_{0}^{1, p}(\omega)$ the closure in $H^{1, p}(\omega)$ of

$$
C_{c}^{\infty}(\omega) \equiv\left\{u \in \bigcap_{m \in \mathbf{N}} C^{m}(\bar{\omega}) \mid \operatorname{supp} u \subset \omega\right\} .
$$

As is customary, $H^{k}(\omega) \equiv H^{k, 2}(\omega)$ and $H_{0}^{1}(\omega) \equiv H_{0}^{1,2}(\omega)$; moreover we write $L^{p} \equiv L^{p}\left(B^{+}\right), H^{k, p} \equiv H^{k, p}\left(B^{+}\right)$and $H_{0}^{1, p} \equiv H_{0}^{1, p}\left(B^{+}\right)$. Finally, for $0 \leq \lambda \leq N+p L^{p, \lambda}(\omega)$ denotes the space of functions $u \in L^{p}(\omega)$ such that

$$
[u]_{L^{p, \lambda}(\omega)}^{p} \equiv \sup _{\substack{y \in \bar{\omega} \\ 0<r \leq \operatorname{diam} \omega}} r^{-\lambda} \inf _{c \in \mathbf{R}} \int_{\omega \cap B_{r}(y)}|u-c|^{p} d x
$$


is finite, normed by

$$
|u|_{L^{p, \lambda}(\omega)} \equiv\left(|u|_{L^{p}(\omega)}^{p}+[u]_{L^{p, \lambda}(\omega)}^{p}\right)^{1 / p}
$$

When $0<\delta \leq 1 L^{p, N+p \delta}(\omega)$ is isomorphic to $C^{0, \delta}(\bar{\omega})$ provided $\partial \omega$ is regular enough [2]. $L^{p, N}(\omega)$ is instead a BMO (acronym of Bounded Mean Oscillation) space: introduced in [8] for $p=1$, it plays a relevant role as a "good substitute" of $L^{\infty}(\omega)$ in PDE's [3], Harmonic Analysis [6], Probability [11]...

Now let $0 \leq \alpha<\infty . C_{\alpha}^{k, \delta}$ denotes the space of functions $u$ such that

$$
|u|_{C_{\alpha}^{k, \delta}} \equiv \sup _{0<s<1} s^{\alpha}|u|_{C^{k, \delta}\left(\overline{B_{[s]}^{+}}\right)}
$$

with $B_{[s]}^{+} \equiv\left\{x \in B^{+} \mid x_{N}>s\right\}$, is finite. (When $\alpha<0$ the right-hand side of (1.1) is finite only for $u=0$.) Of course, $C_{0}^{k, \delta}=C^{k, \delta}\left(B^{+}\right)$. In [7, Lemma 2.1] the continuous imbedding

$$
C_{\alpha+\delta^{\prime}}^{0, \delta^{\prime}} \hookrightarrow C_{\alpha}^{0,0}
$$

is proven for $0<\delta^{\prime} \leq 1$ and $\alpha>0$, then extended as

$$
C_{\alpha+k^{\prime}+\delta^{\prime}}^{k+k^{\prime}, \delta+\delta^{\prime}} \hookrightarrow C_{\alpha}^{k, \delta}
$$

for $k, k^{\prime}$ nonnegative integers, $0 \leq \delta<1,-\delta \leq \delta^{\prime} \leq 1-\delta, k^{\prime}+\delta^{\prime} \geq 0$ and $\alpha \geq 0$, provided that $k+\delta-\alpha$ is not a nonnegative integer. The last restriction is illustrated through one-dimensional example of the function $x \rightarrow x^{h} \log x$, which, whenever $h$ is a nonnegative integer, belongs to all spaces $C_{\alpha}^{k, \delta}$ with $k+\delta-\alpha \leq h$ except $C_{0}^{h, 0}$. This, however, shows only that (1.3) is false when $\delta=\alpha=0$ : what about the nonnegative integer values of $k+\delta-\alpha$ for $\delta+\alpha>0$ ? We shall see below (Lemma 1.4) that they provide no exception to the validity of (1.3); our technique, centered around the function spaces we are going to describe now, will also enable us (Lemma 1.3) to handle the exceptional case $\delta=\alpha=0$.

For $1 \leq p \leq \infty$ let $L_{\alpha}^{p}$ be the space of measurable functions $u: B^{+} \rightarrow \mathbf{R}$ such that $|u|_{L_{\alpha}^{p}} \equiv\left|x_{N}^{\alpha} u\right|_{L^{p}}$ is finite. For $1 \leq p<\infty, N \leq \lambda \leq N+p$ we set

$$
[u]_{L_{\alpha}^{p, \lambda}}^{p} \equiv \sup _{\substack{y \in \overline{B^{+}} \\ 0<r \leq 2}} r^{-\lambda} \inf _{c \in \mathbf{R}} \int_{B^{+} \cap B_{r}(y)} x_{N}^{p \alpha}|u-c|^{p} d x
$$

and define $L_{\alpha}^{p, \lambda}$ as the space of functions $u \in L_{\alpha}^{p}$ with $[u]_{L_{\alpha}^{p, \lambda}}<\infty$, normed by

$$
|u|_{L_{\alpha}^{p, \lambda}} \equiv\left(|u|_{L_{\alpha}^{p}}^{p}+[u]_{L_{\alpha}^{p, \lambda}}^{p}\right)^{1 / p}
$$

Clearly, $L_{0}^{p, \lambda}=L^{p, \lambda}\left(B^{+}\right)$whereas all functions from $L^{p, \lambda}\left(B^{+}\right)$which vanish near $S^{0}$ are in $L_{\alpha}^{p, \lambda}$ whatever $\alpha$. Here we are interested only in $\alpha \geq 0$, which we assume to be the case throughout.

Take $\beta$ in $\left[0, \infty\left[\right.\right.$ and let $\omega \neq \varnothing$ be a bounded open subset of $\mathbf{R}^{N}$ which, in addition, lies in the upper half-space if $\beta>0$. We set

$$
(u)_{\omega, \beta} \equiv\left(\int_{\omega} x_{N}^{\beta} d x\right)^{-1} \int_{\omega} x_{N}^{\beta} u d x
$$


as well as

$$
(\cdot)_{y, r ; \beta} \equiv(\cdot)_{B_{r}(y) ; \beta}, \quad(\cdot)_{y, r,+; \beta} \equiv(\cdot)_{B_{r}^{+}(y) ; \beta} \cdot
$$

Lemma 1.1. If $\beta \geq \alpha$ there exists a constant $C$ such that, whenever $u \in L_{\alpha}^{p}$,

$$
\begin{array}{r}
\int_{B^{+} \cap B_{r}(y)} x_{N}^{p \alpha}\left|u-(u)_{B^{+} \cap B_{r}(y) ; \beta}\right|^{p} d x \\
\leq C \inf _{c \in \mathbf{R}} \int_{B^{+} \cap B_{r}(y)} x_{N}^{p \alpha}|u-c|^{p} d x
\end{array}
$$

for $y \in \overline{B^{+}}$and $0<r \leq 2$.

Proof. Let $\int$ stand for $\int_{B^{+} \cap B_{r}(y)}$. We need only prove the existence of a constant $C$ such that

$$
\int x_{N}^{p \alpha}\left|(u-c)_{B^{+} \cap B_{r}(y) ; \beta}\right|^{p} d x \leq C \int x_{N}^{p \alpha}|u-c|^{p} d x
$$

as $c$ varies in $\mathbf{R}$. But for $p>1$ (the case $p=1$ is only simpler) the left-hand side above is majorized by the product of

$$
I \equiv \int x_{N}^{p \alpha} d x\left(\int x_{N}^{\beta} d x\right)^{-p}\left(\int x_{N}^{(\beta-\alpha) p /(p-1)} d x\right)^{p-1}
$$

and

$$
\int x_{N}^{p \alpha}|u-c|^{p} d x
$$

Since there exist positive constants $C_{1}, C_{2}$ such that, if $t \geq 0$,

$$
C_{1} r^{N+t} \leq \int x_{N}^{t} d x \leq C_{2} r^{N+t} \text { when } y_{N} \leq 2 r
$$

and

$$
C_{1} r^{N} y_{N} t \leq \int x_{N}^{t} d x \leq C_{2} r^{N} y_{N}^{t} \quad \text { when } y_{N}>2 r
$$

we have $I \leq C$, whence the desired result.

Lemma 1.2. For $0<\delta \leq 1 L_{\alpha}^{p, N+p \delta}$ is isomorphic to $C_{\alpha}^{0, \delta}$.

Proof. Let $u \in C_{\alpha}^{0, \delta}$. For $y \in B^{+}$and $0<r \leq 2$ we denote by $\hat{y}$ the top point of $\overline{B^{+} \cap B_{r}(y)}$, which satisfies $\hat{y}_{N}>x_{N}$ whatever $x \in B^{+} \cap B_{r}(y)$. Thus,

$$
x_{N}^{p \alpha}|u(x)-u(\hat{y})|^{p} \in \leq x_{N}^{p \alpha}|u|_{C^{0, \delta}\left(\overline{B^{+}}{ }_{\left(x_{N}\right)}\right)}^{p}|x-\hat{y}|^{p \delta} \leq|u|_{C_{\alpha}^{0, \delta}}^{p}|x-\hat{y}|^{p \delta},
$$

and therefore

$$
\begin{aligned}
& \inf _{c \in \mathbf{R}} \int_{B^{+} \cap B_{r}(y)} x_{N}^{p \alpha}|u-c|^{p} d x \\
& \quad \leq|u|_{C_{\alpha}^{0, \delta}}^{p}(2 r)^{p \delta}\left|B^{+} \cap B_{r}(y)\right| \leq C r^{N+p \delta}|u|_{C_{\alpha}^{0, \delta}}^{p}
\end{aligned}
$$

Moreover,

$$
\int_{B^{+}} x_{N}^{p \alpha}|u|^{p} d x \leq \int_{B^{+}} x_{N}^{p \alpha}|u|_{C^{0}\left(\bar{B}^{+}{ }_{\left(x_{N}\right]}\right)}^{p} d x \leq\left|B^{+}\right||u|_{C_{\alpha}^{0,0}}^{p} .
$$

This proves $C_{\alpha}^{0, \delta} \hookrightarrow L_{\alpha}^{p, N+p \delta}$. 

with

Vice versa, let $u \in L_{\alpha}^{p, N+p \delta}$. For any $\left.s \in\right] 0,1\left[\left.u\right|_{B_{[s]}^{+}}\right.$belongs to $L^{p, N+p \delta}\left(B_{[s]}^{+}\right)$

$$
\begin{aligned}
s^{p \alpha}|u|_{L^{p, N+p \delta\left(B_{[s]}^{+}\right)}}^{p} \leq & \int_{B_{[s]}^{+}} x_{N}^{p \alpha}|u|^{p} d x \\
& +\sup _{\substack{y \in \bar{B}_{[s]}^{+} \\
0<r \leq 2}} r^{-(N+p \delta)} \inf _{c \in \mathbf{R}} \int_{B_{[s]}^{+} \cap B_{r}(y)} x_{N}^{p \alpha}|u-c|^{p} d x \\
\leq & |u|_{L_{\alpha}^{p, N+p \delta}}^{p} .
\end{aligned}
$$

But the regularity of $\partial B_{[s]}^{+}$is sufficient to yield $L^{p, N+p \delta}\left(B_{[s]}^{+}\right) \hookrightarrow C^{0, \delta}\left(\overline{B_{[s]}^{+}}\right)$, indeed even with a constant of injection $C$ independent of $s$ if the latter is, say, $\leq 1 / 2[2]$. Hence,

$$
\sup _{0<s \leq 1 / 2} s^{p \alpha}|u|_{C^{0, \delta}\left(\overline{B_{[s]}^{+}}\right)}^{p} \leq C|u|_{L_{\alpha}^{p, N+p \delta}}^{p}
$$

and the conclusion follows.

We have thus obtained, for $0<\delta \leq 1$, equivalence of the norms $|\cdot|_{C_{\alpha}^{0, \delta}}$ and $|\cdot|_{L_{\alpha}^{p, N+p \delta}}$; the latter becomes instead a (weighted) BMO norm in the limit case $\delta=0$, and this circumstance plays a role in the next result.

Lemma 1.3. For $0 \leq \delta<1$ and $0<\delta^{\prime} \leq 1-\delta$ the continuous imbedding

$$
L_{\alpha+\delta^{\prime}}^{p, N+p\left(\delta+\delta^{\prime}\right)} \hookrightarrow L_{\alpha}^{p, N+p \delta}
$$

holds true.

Proof. We fix $\left.\left.y \in \overline{B^{+}}, r \in\right] 0,2\right]$ and write $\int$ for $\int_{B^{+} \cap B_{r}(y)}$. If $y_{N} \geq 2 r$ then $x_{n}>r$ whenever $x \in B_{r}(y)$, and

$$
\begin{aligned}
& r^{-(N+p \delta)} \inf _{c \in \mathbf{R}} \int x_{N}^{p \alpha}|u-c|^{p} d x \\
& \leq r^{-\left[N+p\left(\delta+\delta^{\prime}\right)\right]} \inf _{c \in \mathbf{R}} \int x_{N}^{p\left(\alpha+\delta^{\prime}\right)}|u-c|^{p} d x \\
& \leq[u]_{L_{\alpha+\delta^{\prime}}^{p, N+p\left(\delta+\delta^{\prime}\right)}}^{p}
\end{aligned}
$$

If instead $0 \leq y_{N}<2 r$ we first assume $\delta^{\prime}<1 / p$. Then, if $\left.q \in\right] 0, \infty[$ is such that $\delta^{\prime} p q /(q-p)<1$, Hölder's inequality yields

$$
\int x_{N}^{p \alpha}|u-c|^{p} d x \leq\left(\int x_{N}^{q\left(\alpha+\delta^{\prime}\right)}|u-c|^{q} d x\right)^{p / q}\left(\int x_{N}^{-\delta^{\prime} p q /(q-p)} d x\right)^{1-p / q}
$$

and the second factor on the right-hand side is

$$
\leq C r^{\left[N-\delta^{\prime} p q /(q-p)\right](1-p / q)}=C r^{N(1-p / q)-\delta^{\prime} p}
$$

because now $B^{+} \cap B_{r}(y) \subset B_{3 r}^{+}(\bar{y}), \bar{y}$ being the projection of $y$ over $S^{0}$. 
Therefore,

$$
\begin{aligned}
& r^{-(N+p \delta)} \inf _{c \in \mathbf{R}} \int x_{N}^{p \alpha}|u-c|^{p} d x \\
& \leq C\left(r^{-\left[N+p \delta-N(1-p / q)+\delta^{\prime} p\right] q / p} \inf _{c \in \mathbf{R}} \int x_{N}^{q\left(\alpha+\delta^{\prime}\right)}|u-c|^{q} d x\right)^{p / q} \\
& \quad=C\left(r^{-\left[N+q\left(\delta+\delta^{\prime}\right)\right]} \inf _{c \in \mathbf{R}} \int x_{N}^{q\left(\alpha+\delta^{\prime}\right)}|u-c|^{q} d x\right)^{p / q} \\
& \leq C[u]_{L_{\alpha+\delta^{\prime}}^{q, N+q\left(\delta+\delta^{\prime}\right)}}^{p}
\end{aligned}
$$

But, since

$$
[u]_{L_{\alpha+\delta^{\prime}}^{q, N+q\left(\delta+\delta^{\prime}\right)}} \leq C|u|_{C_{\alpha+\delta^{\prime}}^{0, \delta+\delta^{\prime}}} \leq C|u|_{L_{\alpha+\delta^{\prime}}^{p, N+p\left(\delta+\delta^{\prime}\right)}}
$$

by Lemma 1.2 , we have proved

$$
[u]_{L_{\alpha}^{p, N+p \delta}} \leq C|u|_{L_{\alpha+\delta^{\prime}}^{p, N+p\left(\delta+\delta^{\prime}\right)}}
$$

On the other hand, for $c=(u)_{0,1 ; \alpha+\delta^{\prime}} \quad($ see $(1.4))$ we have

$$
\begin{aligned}
\int_{B^{+}} x_{N}^{p \alpha}|u|^{p} d x & \leq C\left(\int_{B^{+}} x_{N}^{p \alpha}|u-c|^{p} d x+|c|^{p}\right) \\
& \leq C\left([u]_{L_{\alpha}^{p, N+p \delta}+} \int_{B^{+}} x_{N}^{p\left(\alpha+\delta^{\prime}\right)}|u|^{p} d x\right)
\end{aligned}
$$

by Lemma 1.1 , and finally

$$
|u|_{L_{\alpha}^{p, N+p \delta}} \leq C|u|_{L_{\alpha+\delta^{\prime}}^{p, N+p\left(\delta+\delta^{\prime}\right)}}
$$

There remains only to relinquish the restriction $\delta^{\prime}<1 / p$, which can be done in a finite number of steps.

The imbedding (1.5) for $\delta=0$, i.e.,

$$
L_{\alpha+\delta^{\prime}}^{p, N+p \delta^{\prime}} \hookrightarrow L_{\alpha}^{p, N}
$$

is optimal if $\alpha=0$, as the already mentioned one-dimensional example $x \rightarrow$ $\log x$ clearly shows. If instead $\alpha>0$ then (1.6) is weaker than (1.2).

We can finally extend [7, Lemma 2.1] as follows.

Lemma 1.4. Let $k, k^{\prime}$ be nonnegative integers, $0 \leq \delta<1$ with $\alpha+\delta>0$, $-\delta \leq \delta^{\prime} \leq 1-\delta$ with $k^{\prime}+\delta^{\prime} \geq 0$. Then (1.3) holds true.

Proof. When $k=k^{\prime}=0$ (1.3) is nothing but (1.5) for $\delta>0,(1.2)$ for $\delta=0$ (and $\alpha>0$ ).

If $k^{\prime}=0$ we obtain (1.3) for $k \in \mathbf{N}$ through induction, since $|u|_{C_{\alpha}^{k, \delta}}$ is equivalent to $\sum_{|\mu| \leq 1}\left|D^{\mu} u\right|_{C_{\alpha}^{k-1, \delta}}$ and $|u|_{C_{\alpha+\delta^{\prime}}^{k, \delta+\delta^{\prime}}}$ to

$$
\sum_{|\mu| \leq 1}\left|D^{\mu} u\right|_{C_{\alpha+\delta^{\prime}}^{k-1, \delta+\delta^{\prime}}}
$$

From the above it follows that

$$
C_{\alpha+1+\delta^{\prime}}^{1, \delta+\delta^{\prime}}=C_{\alpha+1-\delta+\delta+\delta^{\prime}}^{1, \delta+\delta^{\prime}} \hookrightarrow C_{\alpha+1-\delta}^{1,0}
$$


558

GIOVANNI MARIA TROIANIELLO

since $\alpha+1-\delta \geq 1-\delta>0$, and also that

$$
C_{\alpha+1-\delta}^{1,0}=C_{\alpha+1-\delta}^{0, \delta+1-\delta} \hookrightarrow C_{\alpha}^{0, \delta},
$$

hence

$$
C_{\alpha+1+\delta^{\prime}}^{1, \delta+\delta^{\prime}} \hookrightarrow C_{\alpha}^{0, \delta} .
$$

Since $|u|_{C_{\alpha+k^{\prime}+\delta^{\prime}}^{k+k^{\prime}, \delta+\delta^{\prime}}}$ is equivalent to

$$
\sum_{|\mu| \leq k+k^{\prime}-1}\left|D^{\mu} u\right|_{C_{\alpha+k^{\prime}+\delta^{\prime}}^{1, \delta+\delta^{\prime}}}
$$

and $|u|_{C_{\alpha+k^{\prime}-1}^{k+k^{\prime}-1, \delta}}$ to

$$
\sum_{|\mu| \leq k+k^{\prime}-1}\left|D^{\mu} u\right|_{C_{\alpha+k^{\prime}-1}^{0, \delta}}
$$

(1.7) yields

$$
C_{\alpha+k^{\prime}+\delta^{\prime}}^{k+k^{\prime}, \delta+\delta^{\prime}} \hookrightarrow C_{\alpha+k^{\prime}-1}^{k+k^{\prime}-1, \delta}
$$

for $k$ nonnegative integer and $k^{\prime} \in \mathbf{N}$, so that in the general case (1.3) follows after a finite number of steps.

2. Preliminary $L^{p}$ estimates

In the present section $p$, unless otherwise specified, is arbitrarily fixed in ]1, $\infty\left[\right.$, and $p \equiv p /(p-1)$; moreover, $y \in \mathbf{R}^{N}$ and $0<d<\infty$. Here and throughout we adopt the summation convention.

Lemma 2.1. Let $g^{1}, \ldots, g^{N}, g$ be given in $L^{p}\left(B_{d}(y)\right)$ and let $v$ be any fundion from $H^{1, p}\left(B_{d}(y)\right)$ such that

$$
\int_{B_{d}(y)} v_{x_{i}} \phi_{x_{i}} d x=\int_{B_{d}(y)}\left(g^{i} \phi_{x_{i}}+g \phi\right) d x \quad \forall \phi \in H_{0}^{1, p^{\prime}}\left(B_{d}(y)\right) .
$$

Then we have

$$
\begin{aligned}
& \int_{B_{\rho}(y)}|\nabla v|^{p} d x \leq C\left(\left(\frac{\rho}{r}\right)^{N} \int_{B_{r}(y)}|\nabla v|^{p} d x\right. \\
& \left.+\sum_{i=1}^{N} \int_{B_{r}(y)}\left|g^{i}\right|^{p} d x+r^{p} \int_{B_{r}(y)}|g|^{p} d x\right)
\end{aligned}
$$

for $0<\rho \leq r \leq d$

with $C=C(p)$, as well as

$$
\begin{aligned}
& \sum_{i=1}^{N} \int_{B_{\rho}(y)}\left|v_{x_{i}}-\left(v_{x_{i}}\right)_{y, \rho ; \beta}\right|^{p} d x \\
& \leq C\left(\left(\frac{\rho}{r}\right)^{N+p} \sum_{i=1}^{N} \int_{B_{r}(y)}\left|v_{x_{i}}-\left(v_{x_{i}}\right)_{y, \rho ; \beta}\right|^{p} d x\right) \\
&+\left(\sum_{i=1}^{N} \int_{B_{r}(y)}\left|g^{i}-\left(g^{i}\right)_{y, r ; \beta}\right|^{p} d x+r^{p} \int_{B_{r}(y)}|g|^{p} d x\right)
\end{aligned}
$$

for $0<\rho \leq r \leq d$ 
if $0 \leq \beta<\infty$ (provided $B_{d}(y)$ lies in the upper half-space when $\beta>0$ ), with $C=C(p, \beta)$.

Proof. The Dirichlet problem

$$
\begin{gathered}
V \in H_{0}^{1, p}\left(B_{r}(y)\right), \\
\int_{B_{r}(y)} V_{x_{i}} \phi_{x_{i}} d x=\int_{B_{r}(y)}\left(g^{i} \phi_{x_{i}}+g \phi\right) d x \quad \forall \phi \in H_{0}^{1, p^{\prime}}\left(B_{r}(y)\right)
\end{gathered}
$$

is uniquely solvable, with norm estimate

$$
\int_{B_{r}(y)}|\nabla V|^{p} d x \leq C\left(\sum_{i=1}^{N} \int_{B_{r}(y)}\left|g^{i}\right|^{p} d x+r^{p} \int_{B_{r}(y)}|g|^{p} d x\right) .
$$

Let $\left.z \equiv v\right|_{B_{r}(y)}-V$, so that

$$
\int_{B_{r}(y)} z_{x_{i}} \phi_{x_{i}} d x=0 \quad \forall \phi \in H_{0}^{1, p^{\prime}}\left(B_{r}(y)\right):
$$

whenever $\eta \in C_{c}^{\infty}\left(B_{r}(y)\right)$ the function $Z \equiv \eta z$ satisfies

$$
\begin{gathered}
Z \in H_{0}^{1, p}\left(B_{r}(y)\right), \\
\int_{B_{r}(y)} Z_{x_{i}} \phi_{x_{i}} d x=\int_{B_{r}(y)}\left(\eta_{x_{i}} z \phi_{x_{i}}-\eta_{x_{i}} z_{x_{i}} \phi\right) d x \quad \forall \phi \in H_{0}^{1, p}\left(B_{r}(y)\right),
\end{gathered}
$$

and therefore $Z \in H^{2, p}\left(B_{r}(y)\right)$ with

$$
|Z|_{H^{2, p}\left(B_{r}(y)\right)} \leq C|z|_{H^{1, p}\left(B_{r}(y)\right)} .
$$

Finally, since we can safely replace $\left.v\right|_{B_{r}(y)}$ by $\left.v\right|_{B_{r}(y)}+$ constant and therefore assume $\int_{B_{r}(y)} z d x=0$, Poincaré's inequality yields

$$
|z|_{H^{1, p}\left(B_{r}(y)\right)} \leq C \sum_{i=1}^{N}\left|z_{x_{i}}\right|_{L^{p}\left(B_{r}(y)\right)} .
$$

These observations suffice to show that the smoothness of $z$ away from $\partial B_{r}(y)$ as well as norm estimates such as

$$
|z|_{H^{k, p\left(B_{r / 2}(y)\right)}} \leq C(k, r) \sum_{i=1}^{N}\left|z_{x_{i}}\right|_{L^{p}\left(B_{r}(y)\right)}
$$

can be obtained through a bootstrap argument. But then by choosing $k$ so large that $H^{k, p}\left(B_{r / 2}(y)\right) \hookrightarrow C^{1}\left(\overline{B_{r / 2}(y)}\right)$ we have

$$
\begin{aligned}
\int_{B_{\rho}(y)}|\nabla z|^{p} d x & \leq C \rho^{N} \sum_{i=1}^{N}\left|z_{x_{i}}\right|_{L^{\infty}\left(B_{r / 2}(y)\right)}^{p} \\
& \leq C(r) \rho^{N} \int_{B_{r}(y)}|\nabla z|^{p} d x
\end{aligned}
$$

for $0<p \leq r / 2$, and a change of variables $x \rightarrow x / r$ shows that

$$
\int_{B_{\rho}(y)}|\nabla z|^{p} d x \leq C\left(\frac{\rho}{r}\right)^{N} \int_{B_{r}(y)}|\nabla z|^{p} d x
$$


for $0<\rho \leq r / 2$, hence also for $0<\rho \leq r$ by simple considerations, with $C=C(p)$. We easily arrive at (2.2) by dint of (2.4) and (2.5).

Passing to (2.3) we notice first of all that

$$
\int_{B_{r}(y)}\left(g^{i}\right)_{y, r ; \beta} \phi_{x_{i}} d x=0 \quad \forall \phi \in H_{0}^{1, p^{\prime}}\left(B_{r}(y)\right),
$$

so that $\left.v\right|_{B_{r}(y)}$ can be written as a sum $V+z$ with

$$
\begin{aligned}
\int_{B_{r}(y)} & |\nabla V|^{p} d x \\
\leq & C\left(\sum_{i=1}^{N} \int_{B_{r}(y)}\left|g^{i}-\left(g^{i}\right)_{y, r ; \beta}\right|^{p} d x+r^{p} \int_{B_{r}(y)}|g|^{p} d x\right)
\end{aligned}
$$

and $\Delta z=0$ in $B_{r}(y)$. But $z^{\prime} \equiv z-x_{i}\left(z_{x_{i}}\right)_{y, r ; \beta}$ satisfies the same equation as $z$ and can be assumed to have a vanishing integral over $B_{r}(y)$, so that

$$
\begin{aligned}
& \sum_{i=1}^{N} \int_{B_{\rho}(y)}\left|z_{x_{i}}-\left(z_{x_{i}}\right)_{y, \rho ; \beta}\right|^{p} d x \\
& \quad=\sum_{i=1}^{N} \int_{B_{\rho}(y)}\left|z_{x_{i}}^{\prime}-\left(z_{x_{i}}^{\prime}\right)_{y, r ; \beta}\right|^{p} d x \\
& \quad \leq C \sum_{i=1}^{N} \int_{B_{\rho}(y)}\left|z_{x_{i}}^{\prime}-z_{x_{i}}^{\prime}(y)\right|^{p} d x \\
& \quad \leq C \rho^{N+p} \sum_{i, j=1}^{N}\left|z_{x_{i} x_{j}}^{\prime}\right|_{L^{\infty}\left(B_{r / 2}(y)\right)}^{p} \\
& \quad \leq C(k, r) \rho^{N+p} \int_{B_{r}(y)}\left|\nabla z^{\prime}\right|^{p} d x
\end{aligned}
$$

for some $0<\rho \leq r / 2$, provided $k$ is so large that $H^{k, p}\left(B_{r / 2}(y)\right) \hookrightarrow C^{2}\left(\overline{B_{r / 2}(y)}\right)$. At this point it is easy to ascertain that

$$
\begin{aligned}
& \sum_{i=1}^{N} \int_{B_{\rho}(y)}\left|z_{x_{i}}-\left(z_{x_{i}}\right)_{y, \rho ; \beta}\right|^{p} d x \\
& \quad \leq C\left(\frac{\rho}{r}\right)^{N+p} \sum_{i=1}^{N} \int_{B_{r}(y)}\left|z_{x_{i}}-\left(z_{x_{i}}\right)_{y, r ; \beta}\right|^{p} d x
\end{aligned}
$$

for $0<\rho \leq r$, and that (2.3) follows from (2.6), (2.7).

Corollary. In addition to the hypotheses of Lemma 2.1 suppose that

$$
\begin{aligned}
& \sum_{i=1}^{N} \int_{B_{r}(y)} x_{N}^{p \beta}\left|g^{i}-\left(g^{i}\right)_{y, r ; \beta}\right|^{p} d x \\
& \quad+r^{p} \int_{B_{r}(y)} x_{N}^{p \beta}|g|^{p} d x \leq K r^{\lambda} \quad \text { for } 0<r \leq d
\end{aligned}
$$


with $K>0,0 \leq \lambda<N+p$ and $\beta$ in $\left[0, \infty\left[\right.\right.$ if $y_{N} / 2 \geq d, \beta=0$ otherwise. Then we have

$$
\begin{aligned}
& \rho^{-\lambda} \sum_{i=1}^{N} \int_{B_{\rho}(y)} x_{N}^{p \beta}\left|v_{x_{i}}-\left(v_{x_{i}}\right)_{y, \rho ; \beta}\right|^{p} d x \\
& \quad \leq C\left(d^{-\lambda} \sum_{i=1}^{N} \int_{B_{d}(y)} x_{N}^{p \beta}\left|v_{x_{i}}-\left(v_{x_{i}}\right)_{y, d ; \beta}\right|^{p} d x+K\right) \quad \text { for } 0<\rho \leq d
\end{aligned}
$$

with $C=C(p, \beta, \lambda)$.

Proof. When $y_{N} / 2 \geq d$ and $\beta>0$ we can freely insert a factor $x_{N}^{p \beta}$ inside all the integrals of (2.3) simultaneously because there exists $k \in \mathbf{N}, k \geq 2$ such that $(k-1) r<x_{N}<(k+2) r$ for $x \in B_{r}(y)$. In all cases we see that, by (2.8), for $0<\rho \leq r \leq d$ the function $\Phi(\rho)$ defined as the left-hand side of (2.9) satisfies

hence also

$$
\Phi(\rho) \leq C\left(\left(\frac{\rho}{r}\right)^{N+p} \Phi(r)+K r^{\lambda}\right)
$$

$$
\Phi(\rho) \leq C\left(\left(\frac{\rho}{r}\right)^{\lambda} \Phi(r)+K \rho^{\lambda}\right)
$$

by a fundamental lemma due to Campanato [2]. The last inequality for $r=d$ is nothing but (2.9).

Lemma 2.2. Take $y_{N}=0$. Let $g^{1}, \ldots, g^{N}$ by given in $L^{p}\left(B_{d}^{+}(y)\right)$ and let $v$ be any function from $H^{1, p}\left(B_{d}^{+}(y)\right)$ such that $v=0$ on $S_{d}^{0}(y)$,

$$
\int_{B_{d}^{+}} v_{x_{i}} \phi_{x_{i}} d x=\int_{B_{d}^{+}(y)} g^{i} \phi_{x_{i}} d x \quad \forall \phi \in H_{0}^{1, p^{\prime}}\left(B_{d}^{+}(y)\right) .
$$

Then we have

$$
\int_{B_{\rho}^{+}(y)}|\nabla v|^{p} d x \leq C\left(\left(\frac{\rho}{r}\right)^{N} \int_{B_{r}^{+}(y)}|\nabla v|^{p} d x+\sum_{i=1}^{N} \int_{B_{r}^{+}(y)}\left|g^{i}\right|^{p} d x\right)
$$

$$
\text { for } 0<\rho \leq r \leq d
$$

with $C=C(p)$, as well as, when $p=2$,

$$
\begin{array}{r}
\sum_{i=1}^{N} \int_{B_{\rho}(y)}\left[v_{x_{i}}-\left(v_{x_{i}}\right)_{y, \rho,+; \beta}\right]^{2} d x \\
\leq C\left(\left(\frac{\rho}{r}\right)^{N+2} \sum_{i=1}^{N} \int_{B_{r}^{+}(y)}\left[v_{x_{i}}-\left(v_{x_{i}}\right)_{y, r,+; \beta}\right]^{2} d x\right. \\
\left.+\sum_{i=1}^{N} \int_{B_{r}^{+}(y)}\left[g^{i}-\left(g^{i}\right)_{y, r,+; \beta}\right]^{2} d x\right) \\
\quad \text { for } 0<\rho \leq r \leq d
\end{array}
$$

if $0 \leq \beta<\infty$, with $C=C(\beta)$.

Proof. If we extend $v$ across $S^{0}$ by setting

$$
\tilde{v}\left(x^{\prime},-x_{N}\right) \equiv-v\left(x^{\prime}, x_{N}\right) \quad \text { for }\left(x^{\prime}, x_{N}\right) \in B_{d}^{+}(y)
$$


and write down the equation satisfied by $\tilde{v},(2.11)$ becomes a straightforward consequence of (2.2).

As for (2.12), we can prove it along the same lines as (2.3): it is worth stressing that, when dealing with $p=2$, we can avail ourselves of the variational theory in $B_{r}^{+}(y)$. See [3].

Corollary. If, in addition to the hypotheses of Theorem 2.2, we suppose that

$$
\sum_{i=1}^{N} \int_{B_{r}^{+}(y)}\left|g^{i}\right|^{p} d x \leq K r^{\lambda} \quad \text { for } 0<r \leq d
$$

with $K>0$ and $0 \leq \lambda<N$, then we have

$$
\rho^{-\lambda} \int_{B_{\rho}^{+}(y)}|\nabla v|^{p} d x \leq C\left(d^{-\lambda} \int_{B_{d}(y)}|\nabla v|^{p} d x+K\right) \quad \text { for } 0<\rho \leq d
$$

with $C=C(p, \lambda)$. If instead, when $p=2$, we suppose that

$$
\sum_{i=1}^{N} \int_{B_{r}^{+}(y)}\left[g^{i}-\left(g^{i}\right)_{y, r,+; \beta}\right]^{2} d x \leq K r^{\lambda} \text { for } 0<r \leq d
$$

with $K>0$ and $0 \leq \lambda<N+2$, then we have

$$
\begin{aligned}
& \rho^{-\lambda} \sum_{i=1}^{N} \int_{B_{\rho}^{+}(y)}\left[v_{x_{i}}-\left(v_{x_{i}}\right)_{y, \rho,+; \beta}\right]^{2} d x \\
& \quad \leq C\left(d^{-\lambda} \sum_{i=1}^{N} \int_{B_{d}^{+}(y)}\left[v_{x_{i}}-\left(v_{x_{i}}\right)_{y, d,+; \beta}\right]^{2} d x+K\right) \quad \text { for } 0<\rho \leq d
\end{aligned}
$$

with $C=C(\beta, \lambda)$.

Proof. Again it suffices to make use of Campanato's Lemma [C1] after introducing auxiliary functions $\Phi(\rho)$ defined as the right-hand side of either (2.14) or (2.16).

Remark. Inspection of the proof of Lemma 2.1 shows that in the right-hand side of (2.2) and (2.3), hence also in the left-hand side of (2.8), each $g^{i}$ can be replaced by any $g_{(r)}^{i} \in L^{p}\left(B_{r}(y)\right)$ such that $g^{i}-g_{(r)}^{i}$ is independent of $x_{i}$. By the same token (2.14) and (2.16) remain valid if each $g^{i}$ in (2.13) and (2.15), respectively, is replaced by any $g_{(r)}^{i} \in L^{p}\left(B_{r}^{+}(y)\right) \quad(p=2$ in the case (2.15)) with $g^{i}-g_{(r)}^{i}$ independent of $x_{i}$.

\section{REGULARITY OF FIRST DERIVATIVES}

We fix some $\delta \in[0,1[$, some $\alpha \in[0,1+\delta[$, and choose $p=2$ if $\alpha \leq \delta$, $p \in] 1,1 /(\alpha-\delta)[$ if $\alpha>\delta$. Let $N+1$ functions

$$
f^{1}, \ldots, f^{N} \in C_{\alpha}^{0, \delta} \text { and } f \in L_{1+\alpha-\delta}^{\infty}
$$

be given.

In all cases the $f^{i}$ 's belong to $L^{p}$. For $\alpha \leq \delta$ and $\delta>0$, indeed, Lemma 1.3 yields the continuous imbedding $C_{\alpha}^{0, \delta} \hookrightarrow L^{2, N+2(\delta-\alpha)}$-which in particular implies

$$
\int_{B_{r}^{+}(y)}\left[f^{i}-\left(f^{i}\right)_{y, r,+; \alpha}\right]^{2} d x \leq C r^{N+2(\delta-\alpha)}\left|f^{i}\right|_{C_{a}^{0, \delta}}^{2}
$$


by Lemma 1.1, if $y \in S^{0}$ and $B_{r}^{+}(y) \subseteq B^{+}$. For $\alpha>\delta$ we have instead $C_{\alpha}^{0, \delta} \hookrightarrow L_{\alpha-\delta}^{\infty}$ (see (1.2)); but $L_{\alpha-\delta}^{\infty} \hookrightarrow L^{p}$, and in particular

$$
\int_{B_{r}^{+}(y)}\left|f^{i}\right|^{p} d x \leq C r^{N+p(\delta-\alpha)}\left|f^{i}\right|_{C_{\alpha}^{0, \delta}}^{p}
$$

for $B_{r}^{+}(y)$ as above, since $p(\delta-\alpha)>-1$.

To deal with $f$ we proceed as follows. If $y \in S^{0}$ and $B_{r}^{+}(y) \subseteq B^{+}$we denote by $E_{y, r} f$ the trivial extension of $\left.f\right|_{B_{r}(y)}$ to $\mathbf{R}^{N} \backslash B_{r}(y)$ and by $P_{y, r} f$ the function

$$
\left(x^{\prime}, x_{N}\right) \mapsto \int_{x_{N}}^{\infty}\left(E_{y, r} f\right)\left(x^{\prime}, t\right) d t .
$$

It is obvious that

$$
\int_{B_{r}^{+}(y)} f \phi d x=\int_{B_{r}^{+}(y)}\left(P_{y, r} f\right) \phi_{x_{N}} d x \quad \forall \phi \in C_{c}^{\infty}\left(B_{r}^{+}(y)\right) ;
$$

moreover,

$$
\begin{aligned}
\int_{B_{r}^{+}(y)}\left|P_{y, r} f\right|^{p} d x & \leq \int_{S_{r}^{0}(y)} d x^{\prime} \int_{0}^{\infty}\left(\int_{x_{N}}^{\infty}\left|\left(E_{y, r} f\right)\left(x^{\prime}, t\right)\right| d t\right)^{p} d x_{N} \\
& \leq C \int_{S_{r}^{0}(y)} d x^{\prime} \int_{0}^{\infty} x_{N}^{p}\left|\left(E_{y, r} f\right)\left(x^{\prime}, x_{N}\right)\right|^{p} d x_{N} \\
& =C \int_{S_{r}^{0}(y)} d x^{\prime} \int_{0}^{\sqrt{r^{2}-\left|x^{\prime}-y^{\prime}\right|^{2}}} x_{N}^{p}\left|f\left(x^{\prime}, x_{N}\right)\right|^{p} d x_{N}
\end{aligned}
$$

(where use has been made of Hardy's inequality [10]), and therefore

$$
\int_{B_{r}^{+}(y)}\left|P_{y, r} f\right|^{p} d x \leq C r^{N+p(\delta-\alpha)}|f|_{L_{1+\alpha-\delta}^{\infty}}^{p} .
$$

Let $u \in H^{1, p}$ satisfy $u=0$ on $S^{0}$,

$$
\int_{B^{+}} u_{x_{i}} \phi_{x_{i}} d x=\int_{B^{+}}\left(f^{i} \phi_{x_{i}}+f \phi\right) d x \quad \forall \phi \in C_{c}^{\infty}\left(B^{+}\right)
$$

and in addition (for simplicity's sake)

$$
\text { supp } \left.u \subseteq \overline{B_{R}^{+}} \text {for some } R \in\right] 0,1[\text {. }
$$

By the $L^{p}$ theory of boundary value problems $u$ satisfies

$$
|u|_{H^{1, p}} \leq C\left(\sum_{i=1}^{N}\left|f^{i}\right|_{C_{\alpha}^{0, \delta}}+|f|_{L_{1+\alpha-\delta}^{\infty}}\right)
$$

(where (3.3)-(3.5) have been utilized with $y=0, r=1$ ). For what concerns regularity we have

Theorem 1. Let $0 \leq \delta<1,0 \leq \alpha<1+\delta$. Under assumption (3.1) all first derivatives of $u$ belong to $L_{\alpha}^{p, N+p \delta}$ with norm estimates

$$
\sum_{i=1}^{N}\left|u_{x_{i}}\right|_{L_{\alpha}^{p, N+p \delta}} \leq C\left(\sum_{i=1}^{N}\left|f^{i}\right|_{C_{\alpha}^{0, \delta^{+}}}|f|_{L_{1+\alpha-\delta}^{\infty}}\right) .
$$


Proof. By (3.7) it suffices to provide estimates over $B^{+} \cap B_{r}(y)$ when $y \in$ ${\overline{B^{+}}}_{(1+R) / 2}$ and, say, $0<r \leq(1-R) / 6$. We proceed in three steps.

Step 1. Interior estimates. Let $y \in \bar{B}_{(1+R) / 2} \backslash{\overline{S^{0}}}_{(1+R) / 2}$ and denote by $d$ the minimum between $y_{N} / 2$ and $(1-R) / 6$. Since $\left.v \equiv u\right|_{B_{d}(y)}$ satisfies (2.1) with $\left.g^{i} \equiv f^{i}\right|_{B_{d}(y)},\left.g \equiv f\right|_{B_{d}(y)}$ and since

$$
\begin{gathered}
\sum_{i=1}^{N} \int_{B_{r}(y)} x_{N}^{p \alpha}\left|f^{i}-\left(f^{i}\right)_{y, r ; \beta}\right|^{p} d x+r^{p} \int_{B_{r}(y)} x_{N}^{p \alpha}|f|^{p} d x \\
\leq C\left(\sum_{i=1}^{N}\left|f^{i}\right|_{C_{\alpha}^{0, \delta}}^{p}+|f|_{L_{1+\alpha-\delta}^{\infty}}^{p}\right) r^{N+p \delta},
\end{gathered}
$$

the corollary to Lemma 2.1 with $\lambda=N+p \delta$ and $\beta=\alpha$ yields

$$
\begin{aligned}
& r^{-(N+p \delta)} \sum_{i=1}^{N} \int_{B_{r}(y)} x_{N}^{p \alpha}\left|u_{x_{i}}-\left(u_{x_{i}}\right)_{y, r ; \alpha}\right|^{p} d x \\
& \leq C\left(d^{-(N+p \delta)} \sum_{i=1}^{N} \int_{B_{d}(y)} x_{N}^{p \alpha}\left|u_{x_{i}}-\left(u_{x_{i}}\right)_{y, d ; \alpha}\right|^{p} d x\right. \\
& \left.+\sum_{i=1}^{N}\left|f^{i}\right|_{C_{\alpha}^{0, \delta}}^{p}+|f|_{L_{1+\alpha-\delta}^{\infty}}^{p}\right) \quad \text { for } 0<r \leq d .
\end{aligned}
$$

If $d=(1-R) / 6(3.8)$ implies

$$
\begin{aligned}
& d^{-(N+p \delta)} \sum_{i=1}^{N} \int_{B_{d}(y)} x_{N}^{p \alpha}\left|u_{x_{i}}-\left(u_{x_{i}}\right)_{y, d ; \alpha}\right|^{p} d x \\
& \leq C \int_{B^{+}}|\nabla u|^{p} d x \leq C\left(\sum_{i=1}^{N}\left|f^{i}\right|_{C_{\alpha}^{0, \delta}}^{p}+|f|_{L_{1+\alpha-\delta}^{\infty}}^{p}\right)
\end{aligned}
$$

and therefore also

$$
\begin{gathered}
r^{-(N+p \delta)} \sum_{i=1}^{N} \int_{B_{r}(y)} x_{N}^{p \alpha}\left|u_{x_{i}}-\left(u_{x_{i}}\right)_{y, r ; \alpha}\right|^{p} d x \\
\leq C\left(\sum_{i=1}^{N}\left|f^{i}\right|_{C_{\alpha}^{0, \delta}}^{p}+|f|_{L_{1+\alpha-\delta}^{\infty}}^{p}\right)
\end{gathered}
$$

for $0<r \leq(1-R) / 6 \leq y_{N} / 2$. that

$$
\begin{aligned}
& r^{-(N+p \delta)} \sum_{i=1}^{N} \int_{B^{+} \cap B_{r}(y)} x_{N}^{p \alpha}\left|u_{x_{i}}-\left(u_{x_{i}}\right)_{B^{+} \cap B_{r}(y) ; \alpha}\right|^{p} d x \\
& \leq C(3 r)^{-(N+p \delta)} \sum_{i=1}^{N} \int_{B_{3 r}^{+}(\bar{y})} x_{N}^{p \alpha}\left|u_{x_{i}}-\left(u_{x_{i}}\right)_{\bar{y}, 3 r,+\alpha}\right|^{p} d x
\end{aligned}
$$


for $0<y_{N} / 2 \leq r \leq(1-R) / 6$, so that

$$
\begin{aligned}
& r^{-(N+p \delta)} \sum_{i=1}^{N} \int_{B_{r}(y)} x_{N}^{p \alpha}\left|u_{x_{i}}-\left(u_{x_{i}}\right)_{y, r ; \alpha}\right|^{p} d x \\
& \leq C\left[(3 d)^{-(N+p \delta)} \sum_{i=1}^{N} \int_{B_{3 d}^{+}(\bar{y})} x_{N}^{p \alpha}\left|u_{x_{i}}-\left(u_{x_{i}}\right)_{\bar{y}, 3 d,+; \alpha}\right|^{p} d x\right. \\
&\left.+\sum_{i=1}^{N}\left|f^{i}\right|_{C_{\alpha}^{0, \delta}}^{p}+|f|_{L_{1+\alpha-\delta}^{\infty}}^{p}\right]
\end{aligned}
$$

for $0<r \leq d=y_{N} / 2<(1-R) / 6$.

We are thus left with the task of giving bounds over hemispheres.

Step 2. Completion of the proof when $\alpha \leq \delta$. Let $y \in{\overline{S^{0}}}_{(1+R) / 2}$ and $r \in$ ]0, $(1-R) / 2$ ] be fixed. Thanks to $(3.4)$, for $d \equiv(1-R) / 2$ the functions $\left.v \equiv u\right|_{B_{d}^{+}(y)}$ satisfies (2.10) with $\left.g^{i} \equiv f^{i}\right|_{B_{d}^{+}(y)}$ if $i=1, \ldots, N-1$ and $g^{N} \equiv$ $\left.\left(f^{N}+P_{y, d} f\right)\right|_{B_{d}^{+}(y)}$. Let $\lambda \equiv N+2(\delta-\alpha), \beta \equiv \alpha$. By (2.16) and the remark at the end of $\S 2$ for what concerns $g^{N}$ it is easy to deduce

$$
\begin{aligned}
& r^{-[N+2(\delta-\alpha)]} \sum_{i=1}^{N} \int_{B_{r}^{+}(y)}\left[u_{x_{i}}-\left(u_{x_{i}}\right)_{y, r,+; \alpha}\right]^{2} d x \\
& \leq C\left(\int_{B^{+}}|\nabla u|^{2} d x+\sum_{i=1}^{N}\left|f^{i}\right|_{C_{\alpha}^{0, \delta}}^{2}+|f|_{L_{1-\alpha-\delta}^{\infty}}^{2}\right) \\
& \text { for } 0<r \leq(1-R) / 2
\end{aligned}
$$

from (3.2) and (3.5). But

$$
r^{-(N+2 \delta)} \sum_{i=1}^{N} \int_{B_{r}^{+}(y)} x_{N}^{2 \alpha}\left[u_{x_{i}}-\left(u_{x_{i}}\right)_{y, r,+, \alpha}\right]^{2} d x \leq \text { 1.h.s. of }(3.13),
$$

so that

$$
\begin{aligned}
& r^{-(N+2 \delta)} \sum_{i=1}^{N} \int_{B_{r}^{+}(y)} x_{N}^{2 \alpha}\left[u_{x_{i}}-\left(u_{x_{i}}\right)_{y, r,+; \alpha}\right]^{2} d x \\
& \leq C\left(\sum_{i=1}^{N}\left|f^{i}\right|_{C^{0, \delta}}^{2}+|f|_{L_{1+\alpha-\delta}^{\infty}}^{2}\right) \quad \text { for } 0<r \leq(1-R) / 2
\end{aligned}
$$

thanks to (3.8). At this point we need only add (3.14) to (3.10)-(3.12) (with $p=2$ ) to obtain the bound on

$$
\sup _{\substack{y \in \bar{B}^{+}(1+R) / 2 \\ 0<r \leq(1-R) / 6}} r^{-(N+2 \delta)} \sum_{i=1}^{N} \sum_{B^{+} \cap B_{r}(y)}^{2 \alpha}\left[u_{x_{i}}-\left(u_{x_{i}}\right)_{B^{+} \cap B_{r}(y) ; \alpha}\right]^{2} d x
$$

as in (3.9).

Step 3. Completion of the proof when $\alpha>\delta$. Again we set $d \equiv(1-R) / 2$ and apply the corollary of Lemma 2.2 (and the remark following it) to $\left.v \equiv u\right|_{B_{d}^{+}(y)}$. 
This time we utilize (2.14) with $\lambda \equiv N+p(\delta-\alpha), \beta \equiv \alpha$, and deduce

$$
\begin{aligned}
& r^{-[N+p(\delta-\alpha)]} \int_{B_{r}^{+}(y)}|\nabla u|^{p} d x \\
& \quad \leq C\left(\sum_{i=1}^{N}\left|f^{i}\right|_{C_{\alpha}^{0, \delta}}^{p}+|f|_{L_{1+\alpha-\delta}^{\infty}}^{p}\right) \text { for } 0<r \leq(1-R) / 2
\end{aligned}
$$

from (3.2), (3.5) and (3.8). But

$$
r^{-(N+p \delta)} \sum_{i=1}^{N} \int_{B_{r}^{+}(y)} x_{N}^{p \alpha}\left|u_{x_{i}}-\left(u_{x_{i}}\right)_{y, r,+; \alpha}\right|^{p} d x
$$

does not exceed the left-hand side of (3.15), and the conclusion follows easily.

\section{REGULARITY OF HIGHER ORDER DERIVATIVES}

In this section we shall "differentiate" (3.6). Since this procedure requires the same regularity assumptions about $f$ as about the distributional derivatives $f_{x_{j}}^{i}$ for $i, j=1, \ldots, N$ we can without loss of generality restrict ourselves to the case $f^{1}=\cdots=f^{N}=0$. This means that we are going to deal with a function $u \in H^{1, p}$ satisfying $u=0$ on $S^{0}$,

$$
\int_{B^{+}} u_{x_{i}} \phi_{x_{i}} d x=\int_{B^{+}} f \phi d x \quad \forall \phi \in C_{c}^{\infty}\left(B^{+}\right)
$$

as well as (3.7). The assumption about $f$ is

$$
f \in C_{\alpha}^{m-2, \delta},
$$

where $m=2,3, \ldots, 0 \leq \delta<1$ and $0 \leq \alpha<m+\delta$. As for $u$, we take it in $H^{1, p}$, where $p$ is chosen as follows: $p=2$ if either $\alpha \leq \delta$ or $\alpha=k+\delta$, and $1<p<1 /(\alpha-k-\delta)$ if $k+\delta<\alpha<k+1+\delta$ for $k=0, \ldots, m-1$.

Lemma 1.4 yields $C_{\alpha}^{m-2, \delta} \hookrightarrow C_{\alpha-k-\delta}^{m-2-k, 0} \hookrightarrow H^{m-2-k, p}$ for $k=0,1, \ldots$, $m-2$ if $k+\delta<\alpha<k+1+\delta$, as well as $C_{k+\delta}^{m-2, \delta} \hookrightarrow C^{m-2-k+1,0} \hookrightarrow H^{m-2-k}$ for $k=1, \ldots, m-2$, since $C_{1}^{1,0} \hookrightarrow L^{2, N} \hookrightarrow L^{2}$ by Lemma 1.3 , and finally $C_{\delta}^{m-2, \delta} \hookrightarrow H^{m-2}$. Let $\alpha \geq m-1+\delta$. When $\nu$ is multi-index of length $|\nu|=m-2$ we have $D^{\nu} f \in L_{\alpha-\delta}^{\infty}$ and in particular

$$
\int_{B_{r}^{+}(y)} x_{N}^{p(m-1)}\left|D^{\nu} f\right|^{p} d x \leq C r^{N+p(m-1+\delta-\alpha)}|f|_{C_{\alpha}^{m-2, \delta}}^{p}
$$

for $y \in S^{0}$ and $B_{r}^{+}(y) \subseteq B^{+}$, since $C_{\alpha}^{m-2, \delta} \hookrightarrow C_{\alpha-\delta}^{m-2,0}$.

Passing to $u$, we need the following weighted $L^{p}$ result (where $H^{1, p^{\prime} ; 0}$ denotes the space of functions in $H^{1, p^{\prime}}$ which vanish on $\left.\partial B^{+} \backslash S^{0}\right)$.

Lemma 4.1. Assume (4.2) with $m-1+\delta \leq \alpha<m+\delta$, where $m=2,3, \ldots$ and $0 \leq \delta<1$. Then, each function $x_{N}^{k-1} D^{\nu} u$ with $|\nu|=k$, where $k=2, \ldots, m$, is in $L^{p}$; each function $U \equiv x_{N}^{m-1} D^{\nu} u_{x_{s}}$, with $|\nu|=m-2, \nu_{N}=0$ and 
$s=1, \ldots, N-1$, vanishes on $S^{0}$ and satisfies

$$
\begin{array}{r}
\int_{B^{+}} U_{x_{i}} \phi_{x_{i}} d x=\int_{B^{+}}\left(\left[-x_{N}^{m-1} D^{\nu} f+\right.\right. \\
\left.+(m-1) x_{N}^{m-2} D^{\nu} u_{x_{N}}\right] \phi_{x_{s}} \\
\left.+(m-1) x_{N}^{m-2} D^{\nu} u_{x_{s}} \phi_{x_{N}}\right) d x \\
\forall \phi \in H^{1, p^{\prime} ; 0},
\end{array}
$$

hence also

$$
\begin{array}{r}
\int_{B^{+}} U_{x_{i}} \phi_{x_{i}} d x=\int_{B^{+}}\left(-x_{N}^{m-1} D^{\nu} f \phi_{x_{s}}+2(m-1) x_{N}^{m-2} D^{\nu} u_{x_{s}} \phi_{x_{N}}\right) d x \\
\forall \phi \in H_{0}^{1, p^{\prime} ; 0} ;
\end{array}
$$

finally,

$$
\sum_{k=1}^{N} \sum_{|\nu|=k} \int_{B^{+}} x_{N}^{p(k-1)}\left|D^{\nu} u\right|^{p} d x \leq C|f|_{C_{\alpha}^{m-2, \delta}}^{p} .
$$

Proof. Let $m=2$. We already know that the functional $\phi \mapsto \int_{B^{+}} f \phi d x$ can be continuously extended from $C_{c}^{\infty}\left(B^{+}\right)$to $H_{0}^{1, p^{\prime}}$ (see the beginning of $\S 3$ ) and that

$$
|u|_{H^{1, p}} \leq C|f|_{C_{\alpha}^{0, \delta}}
$$

(see (3.8)). If $\phi \in H^{1, p^{\prime} ; 0}$ then $x_{N} \phi \in H_{0}^{1, p^{\prime}}$, and (4.1) yields

$$
\int_{B^{+}}\left(x_{N} u\right)_{x_{i}} \phi_{x_{i}} d x=\int_{B^{+}}\left(f x_{N} \phi+u \phi_{x_{N}}-u_{x_{N}} \phi\right) d x .
$$

Since $x_{N} f \in L^{p}, x_{N} u$ belongs to $H^{2, p}$; consequently, $x_{N} u_{x_{s}}$ belongs to $H^{1, p}$, vanishes on $S^{0}$ and satisfies

$$
\int_{B^{+}}\left(x_{N} u_{x_{s}}\right)_{x_{i}} \phi_{x_{i}} d x=\int_{B^{+}}\left[\left(-x_{N} f+u_{x_{N}}\right) \phi_{x_{s}}+u_{x_{s}} \phi_{x_{N}}\right] d x
$$

for $\phi \in H^{1, p^{\prime} ; 0}$, i.e., (4.4) and hence also (4.5) in the case at hand. Thanks to (4.7), the $L^{p}$ theory for (4.8) yields

$$
\sum_{s=1}^{N-1} \int_{B^{+}} x_{N}^{p}\left|\nabla u_{x_{s}}\right|^{p} d x \leq C|f|_{C_{\alpha}^{0, \delta}}^{p}
$$

and (4.6) for $m=2$ follows from (4.7), (4.9) and the identity

$$
u_{x_{N} x_{N}}=-\sum_{i=1}^{N-1} u_{x_{s} x_{s}}-f \text { in } B^{+}
$$

Supposing the theorem valid for some $m \geq 2$, let $f \in C_{\alpha+1}^{m-1, \delta} \hookrightarrow C_{\alpha}^{m-2, \delta}$. 
Then, in particular, $U$ is in $H^{1, p}$ and satisfies (4.4): hence,

$$
\begin{aligned}
\int_{B^{+}}( & \left.x_{N} U\right)_{x_{i}} \phi_{x_{i}} d x \\
= & \int_{B^{+}}\left[U_{x_{i}}\left(x_{N} \phi\right)_{x_{i}}+U \phi_{x_{N}}-U_{x_{N}} \phi\right] d x \\
= & \int_{B^{+}}\left(\left[x_{N}^{m} D^{\nu} f_{x_{s}}-(m-1) x_{N}^{m-1} D^{\nu} u_{x_{s} x_{N}}\right.\right. \\
\quad & \left.\quad+(m-1) x_{N}^{m-2} D^{\nu} u_{x_{s}}-(m-1) x_{N}^{m-2} D^{\nu} u_{x_{s}}-x_{N}^{m-1} D^{\nu} u_{x_{s} x_{N}}\right] \phi \\
& \left.\quad+\left[(m-1) x_{N}^{m-1} D^{\nu} u_{x_{s}}+x_{N}^{m-1} D^{\nu} u_{x_{s}}\right] \phi_{x_{N}}\right) d x \\
= & \int_{B^{+}}\left[\left(x_{N}^{m} D^{\nu} f_{x_{s}}-m x_{N}^{m-1} D^{\nu} u_{x_{s} x_{N}}\right) \phi+m x_{N}^{m-1} D^{\nu} u_{x_{s}} \phi_{x_{N}}\right] d x
\end{aligned}
$$

whenever $\phi$ is a smooth function with support in $B^{+} \cup S^{0}$. But this implies $x_{N} U \in H^{2, p}$ and the validity of (4.4) for $m+1$ instead of $m, D^{\nu} \partial / \partial x_{s}$ instead of $D^{\nu}$ follows after replacement of $\phi$ by $\phi_{x_{t}}, t=1, \ldots, N-1$.

Theorem 2. Let $m \in \mathbf{N}, m \geq 2,0 \leq \delta 1,0 \leq \alpha<m+\delta$. Under assumption (4.2) all mth order derivatives of $u$ belong to $L_{\alpha}^{p, N+p \delta}$ with norm estimate

$$
\sum_{|\mu|=m}\left|D^{\mu} u\right|_{L_{\alpha}^{p, N+p \delta}} \leq C|f|_{C_{\alpha}^{m-2, \delta}} .
$$

Proof. Step 1. The case $\alpha>m-1+\delta$. Let $y \in{\overline{B^{+}}}_{(1+R) / 2} \backslash S_{(1+R) / 2}^{0}$ and

$$
0<r \leq d \equiv \min \left(y_{N} / 2,(1-R) / 6\right) \text {. }
$$

By the $L^{p}$ theory, for $|\nu|=m-2$ and $s=1, \ldots, N$, the function $\left.D^{\nu} u_{x_{s}}\right|_{B_{d}(y)}$ is in $H^{1, p}\left(B_{d}(y)\right)$ and satisfies

$$
\int_{B_{d}(y)}\left(D^{\nu} u_{x_{s}}\right)_{x_{i}} \phi_{x_{i}} d x=-\int_{B_{d}(y)}\left(D^{\nu} f\right) \phi_{x_{s}} d x \quad \forall \phi \in H_{0}^{1, p^{\prime}}\left(B_{d}(y)\right),
$$

so that we can proceed as in Step 1 of the proof of Theorem 1 and arrive at an estimate

$$
\begin{array}{r}
r^{-(N+p \delta)} \sum_{|\mu|=m} \int_{B_{r}(y)} x_{N}^{p \alpha}\left|D^{\mu} u-\left(D^{\mu} u\right)_{y, r ; \alpha}\right|^{p} d x \\
\leq C\left(d^{-(N+p \delta)} \sum_{|\mu|=m} \int_{B_{d}(y)} x_{N}^{p \alpha}\left|D^{\mu} u-\left(D^{\mu} u\right)_{y, d ; \alpha}\right|^{p} d x+|f|_{C_{\alpha}^{m-2, \delta}}^{p}\right) \\
\text { for } 0<r \leq d .
\end{array}
$$

If $d=(1-R) / 6$ we bound the right-hand side above with

$$
C\left(\sum_{|\mu|=m} \int_{B^{+}} x_{N}^{p(m-1)}\left|D^{\mu} u\right|^{p} d x+|f|_{C_{a}^{m-2, \delta}}^{p}\right) \leq C|f|_{C_{a}^{m-2, \delta}}^{p}
$$

(see (4.6)), so that we are left with the task of bounding functions

$$
r \mapsto \int_{B_{r}^{+}(y)} x_{N}^{p \alpha}\left|D^{\mu} u-\left(D^{\mu} u\right)_{y, r,+; \alpha}\right|^{p} d x
$$

as $y$ varies in ${\overline{S^{0}}}_{(1+R) / 2}$ and $r$ in $] 0,(1-R) / 2[$. 
To do this we fix $\nu$ with $|\nu|=m-2$ and $\nu_{N}=0$, as well as $s=1, \ldots, N-$ 1. Suppose we know that

$$
\int_{B_{r}^{+}(y)} x_{N}^{p(m-2)}\left|\nabla D^{\nu} u\right|^{p} d x \leq C r^{N+\rho(m-1+\delta-\alpha)}|f|_{C_{\alpha}^{m-2, \delta}}^{p}
$$

for $0<r \leq(1-R) / 2$ (which is the case when $m=2$, indeed even with $|f|_{L_{\alpha-\delta}^{\infty}}$ instead of $|f|_{C_{\alpha}^{0, \delta}}$ : see (3.15)). Thanks to (4.3) and (4.12) we can tackle (4.5) in the light of the corollary to Lemma 2.2, thus obtaining (see (2.14))

$$
r^{-[N+p(m-1+\delta-\alpha)]} \int_{B_{r}^{+}(y)}|\nabla U|^{p} d x \leq C|f|_{C_{a}^{m-2, \delta}}^{p} \text { for } 0<r \leq(1-R) / 2
$$

after utilizing (4.6) to bound $\int_{B^{+}}|\nabla U|^{p} d x$.

From (4.13) we first of all deduce that, whenever $f \in C_{\alpha+1}^{m-1, \delta} \hookrightarrow C_{\alpha}^{m-2, \delta}$, (4.12) holds with $m$ replaced by $m+1, \alpha$ by $\alpha+1$ and $D^{\nu}$ by $D^{\nu} \partial / \partial x_{s}$ : this means that (4.12) has been inductively proven for all values of $m$. Next, since

$$
\begin{gathered}
r^{-(N+p \delta)} \sum_{i=1}^{N} \int_{B_{r}^{+}(y)} x_{N}^{p \alpha}\left|D^{\nu} u_{x_{s} x_{i}}-\left(D^{\nu} u_{x_{s} x_{i}}\right)_{y, r,+; \alpha}\right|^{p} d x \\
\leq r^{-[N+p(m-1+\delta-\alpha)]} \int_{B_{r}^{+}(y)} x_{N}^{p(m-1)}\left|\nabla D^{\nu} u_{x_{s}}\right|^{p} d x
\end{gathered}
$$

and

$$
\begin{aligned}
& \int_{B_{r}^{+}(y)} x_{N}^{p(m-1)}\left|\nabla D^{\nu} u_{x_{s}}\right|^{p} d x \\
& \quad \leq C\left(\int_{B_{r}^{+}(y)}|\nabla U|^{p} d x+\int_{B_{r}^{+}(y)} x_{N}^{p(m-2)}\left|D^{\nu} u_{x_{s}}\right|^{p} d x\right),
\end{aligned}
$$

(4.12) and (4.13) yield the required bound for all derivatives $D^{\nu} u_{x_{s} x_{i}}$ with $\nu, s$ as above and $i=1, \ldots, N$, hence for all $m$ th order derivatives.

Step 2. The case $\alpha=m-1+\delta$. If $y \in{\overline{B^{+}}}_{(1+R) / 2} \backslash{\overline{S^{0}}}_{(1+R) / 2}$ and $0<r \leq d \equiv$ $\min \left(y_{N} / 2,(1-R) / 6\right)$ we still have $(4.11)(p=2)$.

Let $y \in{\overline{S^{0}}}_{(1+R) / 2}$ and $0<r \leq(1-R) / 2$. Beginning with $m=2$ we recall that, since $C_{1+\delta}^{0, \delta} \hookrightarrow L_{1}^{\infty}$, Theorem 1 yields

$$
\sum_{i=1}^{N} \int_{B_{r}^{+}(y)}\left|u_{x_{i}}-\left(u_{x_{i}}\right)_{y, r,+; 0}\right|^{2} d x \leq C|f|_{C_{1+\delta}^{0, \delta}}^{2} r^{N} .
$$

Together with (4.3) for the case at hand (4.14) enables us to apply the corollary of Lemma 2.2 for (4.8), i.e. (4.4), and obtain a bound on quantities such as $r^{-N} \inf _{c \in \mathbf{R}} \int_{B_{r}^{+}(y)}\left(U_{x_{i}}-c\right)^{2} d x$ or, equivalently, as

$$
r^{-N} \inf _{c \in \mathbf{R}} \int_{B_{r}^{+}(y)}\left(x_{N} u_{x_{s} x_{i}}-c\right)^{2} d x
$$

but this is insufficient for our purposes because we need instead to bound quantities such as $r^{-N} \inf _{c \in \mathbf{R}} \int_{B_{r}^{+}(y)} x_{N}^{2}\left(u_{x_{s} x_{i}}-c\right)^{2} d x$. To bypass this obstacle we extend (4.4) and (4.5) to equations throughout $B$ as follows. 
First of all, for $y \in S^{0}, 0<r \leq 1$ and $B_{r}^{+}(y) \subseteq B^{+}$we pick up the functions $P_{y, r} f$ of $\xi 3$ and extend them across $S^{0}$, as follows:

$$
\left(\widetilde{P_{y, r} f}\right)\left(x^{\prime},-x_{N}\right) \equiv\left(P_{y, r} f\right)\left(x^{\prime}, x_{N}\right) \quad \text { for }\left(x^{\prime}, x_{N}\right) \in B^{+} .
$$

Then (see (3.5)),

$$
\int_{B_{r}(y)}\left(\widetilde{P_{y, r} f}\right)^{2} d x \leq C|f|_{C_{1+\delta}^{0, \delta}}^{2} r^{N}
$$

moreover (see (3.4)), the extension of $u$ across $S$ defined by

$$
\tilde{u}\left(x^{\prime},-x_{N}\right) \equiv-u\left(x^{\prime}, x_{N}\right) \quad \text { for }\left(x^{\prime}, x_{N}\right) \in B^{+}
$$

satisfies

$$
\int_{B_{r}(y)} \tilde{u}_{x_{i}} \phi_{x_{i}} d x=\int_{B_{r}(y)}\left(\widetilde{P_{y, r} f}\right) \phi_{x_{N}} d x \quad \forall \phi \in C_{c}^{\infty}\left(B_{r}(y)\right)
$$

when restricted to $B_{r}(y)$. Let $y \in{\overline{S^{0}}}_{(1+R) / 2}$ and $0<r \leq d \equiv(1-R) / 2$ : by the remark at the end of $\S 2,(4.15)$ enables us to apply the corollary of Lemma 2.1 and obtain

$$
\sum_{i=1}^{N} \int_{B_{r}(y)}\left[\tilde{u}_{x_{i}}-\left(\tilde{u}_{x_{i}}\right)_{y, r ; 0}\right]^{2} d x \leq C|f|_{C_{1+\delta}^{0, \delta}}^{2} r^{N}
$$

Now let $F^{s} \equiv-x_{N} f+u_{x_{N}}, F^{N} \equiv u_{x_{s}}$, and let $\widetilde{U}, \widetilde{F}^{s}, \widetilde{F}^{N}$ be the respective extensions of $U, F^{s}, F^{N}$ across $S$ defined by

$$
\begin{gathered}
\tilde{U}\left(x^{\prime},-x_{N}\right) \equiv U\left(x^{\prime}, x_{N}\right), \quad \widetilde{F}^{s}\left(x^{\prime},-x_{N}\right) \equiv F^{s}\left(x^{\prime}, x_{N}\right), \\
\widetilde{F}^{N}\left(x^{\prime},-x_{N}\right) \equiv-F^{N}\left(x^{\prime}, x_{N}\right) \text { for }\left(x^{\prime}, x_{N}\right) \in B^{+} .
\end{gathered}
$$

Then we have

$$
\sum_{i=s, N} \int_{B_{r}(y)}\left[\widetilde{F}^{i}-\left(\widetilde{F}^{i}\right)_{y, r ; 0}\right]^{2} d x \leq C|f|_{C_{1+\delta}^{0, \delta}}^{2} r^{N}
$$

by (4.3) and (4.16). But (4.4) implies that $\widetilde{U}$ is a function from $H_{0}^{1}(B)$ satisfying

$$
\int_{B} \widetilde{U}_{x_{i}} \phi_{x_{i}} d x=\sum_{i=s, N} \int_{B} \widetilde{F}^{i} \phi_{x_{i}} d x \quad \forall \phi \in C_{c}^{\infty}(B)
$$

and (4.18) enables us to apply the corollary to Lemma $2.1:^{a}$ thus, a uniform bound

$$
r^{-N} \sum_{i=1}^{N} \int_{B_{r}(y)}\left[\widetilde{U}_{x_{i}}-\left(\widetilde{U}_{x_{i}}\right)_{y, r ; 0}\right]^{2} d x \leq C|f|_{C_{1+\delta}^{0, \delta}}^{2}
$$

easily follows, once the bound

$$
\int_{B^{+}}|\nabla \tilde{U}|^{2} d x \leq C|f|_{C_{1+\delta}^{0, \delta}}^{2}
$$


(see (4.6)) has been taken into account. At this point we observe that $\left(\tilde{u}_{x_{s}}\right)_{y, r ; 0}$ $=\left(\widetilde{U}_{x_{N}}\right)_{y, r, 0}=0$, so that $(4.16)$ and $(4.20)$ yield

$$
\begin{aligned}
r^{-N} \inf _{c \in \mathbf{R}} \int_{B_{r}^{+}(y)} x_{N}^{2}\left(u_{x_{s} x_{N}}-c\right)^{2} d x \\
\quad \leq r^{-N} \int_{B_{r}^{+}(y)}\left(U_{x_{N}}-u_{x_{s}}\right)^{2} d x \\
\quad \leq 2 r^{-N} \int_{B_{r}(y)}\left(\tilde{U}_{x_{N}}^{2}+\tilde{u}_{x_{s}}^{2}\right) d x \leq C|f|_{C_{1+\delta}^{0, \delta}}^{2} .
\end{aligned}
$$

Next, we pass to another extension $\tilde{u}$ of $u$ across $S^{0}$, that is,

$$
\tilde{u}\left(x^{\prime},-x_{N}\right) \equiv u\left(x^{\prime}, x_{N}\right) \quad \text { for }\left(x^{\prime}, x_{N}\right) \in B^{+} \text {: }
$$

a simple argument shows that (4.14) implies

$$
\sum_{i=1}^{N-1} \int_{B_{r}(y)}\left[\tilde{u}_{x_{i}}-\left(\tilde{u}_{x_{i}}\right)_{y, r ; 0}\right]^{2} d x \leq C|f|_{C_{1+\delta}^{0, \delta}}^{2} r^{N} .
$$

Then we let $F^{s} \equiv-x_{N} f, F^{N} \equiv 2 u_{x_{s}}$, and denote by $\widetilde{U}, \widetilde{F}^{s}, \widetilde{F}^{N}$ the respective extensions of $U, F^{s}, F^{N}$ across $S^{0}$ defined by

$$
\begin{gathered}
\widetilde{U}\left(x^{\prime},-x_{N}\right) \equiv-U\left(x^{\prime}, x_{N}\right), \quad \widetilde{F}^{s}\left(x^{\prime},-x_{N}\right) \equiv-F^{s}\left(x^{\prime}, x_{N}\right), \\
\widetilde{F}^{N}\left(x^{\prime},-x_{N}\right) \equiv F^{N}\left(x^{\prime}, x_{N}\right) \quad \text { for }\left(x^{\prime}, x_{N}\right) \in B^{+}
\end{gathered}
$$

again, (4.18) holds (this time by (4.22) in addition to (4.3)), and (4.19) is satisfied (this time as a consequence of (4.5)). By the corollary to Lemma 2.1 (4.20) is still valid. In the present situation, $\left(\widetilde{U}_{x_{i}}\right)_{y, r ; 0}=0$ for $i=1, \ldots, N-$ 1 , so that

$$
\begin{aligned}
& r^{-N} \sum_{i=1}^{N-1} \inf _{c_{i} \in \mathbf{R}} \int_{B_{r}^{+}(y)} x_{N}^{2}\left(u_{x_{s} x_{i}}-c_{i}\right)^{2} d x \\
& \quad \leq r^{-N} \sum_{i=1}^{N-1} \int_{B_{r}(y)} \widetilde{U}_{x_{i}}^{2} d x \leq C|f|_{C_{1+\delta}^{0, \delta}}^{2} .
\end{aligned}
$$

The required bound for all derivatives $u_{x_{s} x_{i}}$ with $s=1, \ldots, N-1$ and $i=1, \ldots, N$, hence for all second derivatives altogether, are consequent on (4.21) and (4.24): we need indeed only remark that

$$
\begin{array}{r}
r^{-(N+2 \delta)} \int_{B_{r}^{+}(y)} x_{N}^{2(1+\delta)}\left[u_{x_{i} x_{j}}-\left(u_{x_{i} x_{j}}\right)_{y, r,+; 1+\delta}\right]^{2} d x \\
\leq r^{-N} \int_{B_{r}^{+}(y)} x_{N}^{2}\left[u_{x_{i} x_{j}}-\left(u_{x_{i} x_{j}}\right)_{y, r,+; 1+\delta}\right]^{2} d x .
\end{array}
$$

An inductive argument shows that for $m>2$ the inequality

$$
r^{-N} \sum_{i=1}^{N} \int_{B_{r}(y)}\left[\widetilde{U}_{x_{i}}-\left(\widetilde{U}_{x_{i}}\right)_{y, r ; 0}\right]^{2} d x \leq C|f|_{C_{m-1+\delta}^{m-2, \delta}}^{2}
$$

(with $U \equiv x_{N}^{m-1} D^{\nu} u_{x_{s}}$ ) is valid as a consequence of (4.19) in either case (4.17) (with $F^{s} \equiv-x_{N}^{m-1} D^{\nu} f+(m-1) x_{N}^{m-2} D^{\nu} u_{x_{N}}, F^{N} \equiv(m-1) x_{N}^{m-2} D^{\nu} u_{x_{s}}$ ) and 
(4.23) (with $\left.F^{s} \equiv-x_{N}^{m-1} D^{\nu} f, F^{N} \equiv 2(m-1) x_{N}^{m-2} D^{\nu} u_{x_{s}}\right)$. We can therefore reach the conclusion of the theorem in the present case by generalizing the proofs of (4.21) and (4.24).

Step 3. The case $\alpha<m+\delta$. Let $s=1, \ldots, N-1: u_{x_{s}}$ is in $H^{2, p}$ and vanishes on $S^{0}$. If $m=2$, we observe that

$$
\int_{B^{+}} u_{x_{s} x_{i}} \phi_{x_{i}} d x=-\int_{B^{+}} f \phi_{x_{s}} d x \quad \forall \phi \in C_{c}^{\infty}\left(B^{+}\right),
$$

and the conclusion follows from Theorem 1. If $m>2$ we write

$$
\int_{B^{+}} u_{x_{s} x_{i}} \phi_{x_{i}} d x=\int_{B^{+}} f_{x_{s}} \phi d x \quad \forall \phi \in C_{c}^{\infty}\left(B^{+}\right):
$$

since $f_{x_{s}} \in C_{\alpha}^{m-3, \delta}$, we proceed by induction (with the help of the two previous steps if $\alpha \geq m-2+\delta)$.

Remark 1 . When $u$ is a sufficiently smooth function satisfying $-\Delta u=f$ in $B^{+}$as well as $u(x)=0$ if $x_{N}=0$ or $|x|>R,(4.10)$ is the result proven in [7, Theorem 3.1] under the additional assumptions $\delta>0$ and $m+\delta-\alpha \notin \mathbf{N}$ (see $(0.3))$.

Remark 2. When assumptions (3.7) is dispensed with, minor modifications in the proofs of Theorems 1 and 2 are needed to yield regularity and norm estimates for derivatives of $\left.u\right|_{B_{R}^{+}}$whatever $\left.R \in\right] 0,1$ [. This means in particular that Theorem 1 with $\alpha=0$ (which is then, basically, a result of [3]) can be considered sufficient for the following theorem to apply. If $Q$ is an $N$-dimensional cube, any continuous linear map from $L^{2}$ into $L^{\infty}(Q)$ which is continuous from $L$ into $L^{2, N}(Q)$ is also continuous from $L^{p}$ into $L^{p}(Q), 2<p<\infty$ $($ see $[5,4])$. Some rather standard techniques can at this point be utilized to proceed from here to the general $L^{p}$ theory of the Dirichlet problem, the range $1<p<2$ being attained through a duality argument (see [2]).

Summing up. Although our results of $\S \S 2-4$ depend heavily on the $L^{p}$ theory for $1<p<\infty$, on strictly logical ground the $L^{2}$ theory is the only prerequisite.

Note that the case $\delta=0$ in Theorems 1 and 2 yields a weighted version of the $L^{\infty} \rightarrow$ BMO type of regularity, whose role in interpolation when $\alpha=0$ has just been hinted at.

Remark 3. Consider a solution $u \in H^{1}$ to the degenerate equation $-x_{N} \Delta u=f$ in $B^{+}$, where $f$ is given in $L^{\infty}$. If $u=0$ on $S^{0}$ and $\operatorname{supp} u \subset B^{+} \cup$ $S^{0}$, Theorems 1 and 2 apply with $f$ replaced by $x_{N}^{-1} f \in L_{1}^{\infty}$. Thus, all first derivatives of $u$ belong to $L_{\delta}^{2, N+2 \delta}$ whatever $\delta \in[0,1]$ and all second derivatives to $L_{1}^{2, N}$, with norm estimates. This result is optimal, as the onedimensional example $u(x) \equiv x \log x$ clearly shows.

(For a thorough investigation of degenerate equations in weighted Hölder function spaces see [1].)

\section{REFERENCES}

1. P. Bolley, J. Camus, and G. Métivier, Estimations de Schauder et régularité hölderienne pour une classe de problèmes aux limites singuliers, Comm. Partial Differential Equations 11 (1986), 1135-1203. 
2. S. Campanato, Proprietà di Hölderianità di alcune classi di funzioni, Ann. Scuola Norm. Sup. Pisa Sci. Fis. Mat. 17 (1963), 175-188.

3. __ Equazioni ellittiche del secondo ordine e spazi $L^{2, \lambda}$, Ann. Mat. Pura Appl. 69 (1965), 321-382.

4. __ Su un teorema di interpolazione di G. Stampacchia, Ann. Scuola Norm Sup. Pisa Sci. Fis. Mat. 20 (1966), 649-652.

5. S. Campanato and G. Stampacchia, Sulle maggiorazioni $L^{p}$ nella teoria delle equazioni ellittiche, Boll. Un. Mat. Ital. 20 (1965), 393-399.

6. C. Fefferman and E. Stein, $H^{p}$ spaces of several variables, Acta Math. 129 (1972), 137-193.

7. D. Gilbarg and L. Hörmander, Intermediate Schauder estimates, Arch. Rational Mech. Anal. 74 (1980), 297-318.

8. F. John and L. Nirenberg, On functions of bounded mean oscillation, Comm. Pure Appl. Math. 14 (1961), 415-426.

9. G. M. Lieberman, Intermediate Schauder estimates for oblique derivative problems, Arch. Rational Mech. Anal. 93 (1986), 129-134.

10. J. Nečas, Les méthodes directs en théorie des équations elliptiques, Masson, Paris, 1967.

11. D. W. Stroock and S. R. J. Varadhan, Multidimensional diffusion processes, Springer, Berlin, Heidelberg, and New York, 1979.

12. G. M. Troianiello, Elliptic differential equations and obstacle problems, Plenum, New York, 1987.

13. _ _ A class of weighted function spaces and intermediate Caccioppoli-Schauder estimates, Journées Équations aux Dérivées Partielles, St.-Jean-de-Monts, 1988.

Dipartimento di Matematica, Università di Roma, Città Universitaria, 00185 Roma, ITALIA 